\title{
Miquel dynamics, Clifford lattices and the Dimer model
}

\author{
Niklas C. Affolter ${ }^{1}$ (C) \\ Received: 18 January 2019 / Revised: 16 March 2021 / Accepted: 21 April 2021 \\ (c) The Author(s) 2021
}

\begin{abstract}
Miquel dynamics was introduced by Ramassamy as a discrete time evolution of square grid circle patterns on the torus. In each time step every second circle in the pattern is replaced with a new one by employing Miquel's six circle theorem. Inspired by this dynamics we consider the local Miquel move, which changes the combinatorics and geometry of a circle pattern. We prove that the circle centers under Miquel dynamics are Clifford lattices, an integrable system considered by Konopelchenko and Schief. Clifford lattices have the combinatorics of an octahedral lattice, and every octahedron contains six intersection points of Clifford's four circle configuration. The Clifford move replaces one of these circle intersection points with the opposite one. We establish a new connection between circle patterns and the dimer model: If the distances between circle centers are interpreted as edge weights, the Miquel move preserves probabilities in the sense of urban renewal.
\end{abstract}

Keywords Miquel dynamics · Circle patterns · Clifford lattices · Dimer model · Urban renewal

\section{Introduction}

Miquel dynamics was first introduced by Ramassamy following an idea of Kenyon, see [11] and references therein. In each time step, this discrete dynamical system replaces every second circle of a square grid circle pattern. If the circle pattern is doubly periodic, it is conjectured that these dynamics feature a form of discrete integrability and that they are related to dimer statistics or dimer integrable systems [4]. First progress toward integrability has been made by Glutsyuk and Ramassamy in [5] for the case of the doubly periodic $2 \times 2$ grid.

In Theorem 3.1 we show that the collection of circle centers under Miquel dynamics form a special case of Clifford lattices, a discrete integrable system studied by

\footnotetext{
$凶$ Niklas C. Affolter

affolter@math.tu-berlin.de

1 TU Berlin, Institute of Mathematics, Strasse des 17. Juni 136, 10623 Berlin, Germany
} 
Konopelchenko and Schief [8]. We introduce the geometric star-ratio function, and the centers of circle patterns are exactly the Clifford lattices with real star-ratios. If the star-ratios are real and positive, we say a circle pattern is Kasteleyn. To a Kasteleyn circle pattern we associate a dimer model with edge weights equal to the distances of circle centers. We show in Theorem 3.4 that the Miquel move induces urban renewal on the associated dimer model. This proves that under Miquel dynamics the star-ratios transform exactly as the face weight coordinates of the discrete cluster integrable system as introduced by Goncharov and Kenyon [4]. We want to stress that all our proofs are of a local nature. This has the advantage that the theory is not restricted to $\mathbb{Z}^{2}$ combinatorics.

Very recently a preprint by Kenyon et al. [7] has appeared that also shows how to realize circle patterns with given star-ratios.

The structure of the paper is as follows: In Sect. 2 we revisit the definition of dimer statistics and introduce notation for the graphs, circle patterns and the starratios considered throughout this paper. We also define both the local Miquel and the local Clifford move. After stating the two main Theorems 3.1 and 3.4 in Sect. 3 we introduce in Sect. 4 the Möbius geometric mutation map which underlies both the Clifford and the Miquel move. We also calculate the transformation formulas for starratios under the Möbius mutation map. In Sect. 5 we study the Clifford configuration, giving a geometric construction of the Möbius mutation map and relating it to work of Konopelchenko and Schief [8]. Additionally, we derive several useful lemmas by investigating the relation between the Clifford configuration, integrable cross ratio systems and integrable circle patterns as defined by Bobenko, Mercat and Suris [2]. Finally, we assemble the pieces in Sect. 6 to relate star-ratios with the Miquel move thereby proving the two main theorems. Even though we prove everything locally, it is interesting how this translates to the setting of lattice dynamics, which we briefly outline in Sect. 7.

\section{Preliminaries}

\subsection{Dimer statistics}

Given a graph $G=(V, E)$ we call a function $\omega: E \rightarrow \mathbb{R}_{+}$an edge weight function, where $\mathbb{R}_{+}$is the set of strictly positive reals. Equivalently, we write $\omega \in \mathbb{R}_{+}^{E}$ and call $\omega$ the edge weights. A simple graph is a graph without loops or multi-edges.

Definition 2.1 A perfect matching of a simple graph $G=(V, E)$ is a subset $M \subset E$ of the edge set such that each vertex of the graph is incident to exactly one edge in $M$. We denote the set of perfect matchings of a graph by $\mathcal{M}(G)$. The weight $\omega(M)$ of a perfect matching $M$ with respect to edge weights $\omega$ is:

$$
\omega(M)=\prod_{e \in M} \omega(e)
$$


The dimer partition function $Z_{G}$ is defined as:

$$
Z_{G}: \mathbb{R}_{+}^{E} \rightarrow \mathbb{R}_{+}, \quad \omega \mapsto \sum_{M \in \mathcal{M}(G)} \omega(M)
$$

The probability $P_{\omega}(M)$ of a perfect matching is proportional to its weight with the partition function as normalization constant:

$$
P_{\omega}(M)=\frac{\omega(M)}{Z_{G}(\omega)}
$$

\subsection{Bipartite surface graphs $\mathcal{G}$}

We start by defining the class $\mathcal{G}$ of bipartite surface graphs for which we prove our lemmas and theorems.

Definition 2.2 A graph $G=(V, E)$ embedded in an oriented and closed surface is in the class of bipartite surface graphs $\mathcal{G}$ if:

- The complement of $G$ is a collection $F$ of disjoint open disks, and $(V, E, F)$ is a locally finite $\mathrm{CW}$-decomposition of the surface.

- $G$ is bipartite, that is the set of vertices $V$ is the disjoint union of the two independent sets $V^{+}$and $V^{-}$.

- Every vertex in $V$ has degree at least 3 and every face has degree at least 2.

In order to simplify notation we assume that we can identify an edge $e \in E$ with its two incident vertices $v$ and $v^{\prime}$ and therefore write $e=\left(v, v^{\prime}\right)$. Similarly for dual edges $e^{*} \in E^{*}$ we write $e^{*}=\left(f, f^{\prime}\right)$ where $f$ and $f^{\prime}$ are the two faces incident to $e$. We usually consider edges at some distinct face $f_{0}$, and the cyclic order of edges around $f_{0}$ will usually clear up any confusion.

We also use a standard orientation of the edges of both $G$ and the dual $G^{*}$. An edge $e=\left(v^{-}, v^{+}\right)$is always incident to a vertex $v^{+} \in V^{+}$and a vertex $v^{-} \in V^{-}$, and we orient that edge as pointing from $v^{-}$to $v^{+}$. Each dual edge $e^{*}$ is oriented such that it crosses the oriented primal edge $e$ from the left to the right. As a consequence, the dual edges are oriented counter clockwise around vertices in $V^{+}$and clockwise around vertices in $V^{-}$. For an example of the standard orientation see Fig. 1.

Definition 2.3 Let $G \in \mathcal{G}$ and let $f \in F$ be a quadrilateral with the four neighbors $f_{1}, f_{2}, f_{3}, f_{4}$. Then we define the edge neighborhood $N_{f}$ of $f$ as the set of edges that are not incident with any other face except $f_{1}, f_{2}, f_{3}, f_{4}$ or $f$. The dual edge neighborhood $N_{f}^{*}$ is the set of edges dual to edges in $N_{f}$.

Definition 2.4 Given $G \in \mathcal{G}$ and a quadrilateral $f \in F$ we denote by mut $f \in \mathcal{G}$ the graph resulting from the 4-mutation at $f$. It differs from $G$ by a local change of combinatorics centered at $f$. We will write $\tilde{G}=\operatorname{mut}_{f} G$ and $\tilde{G}=(\tilde{V}, \tilde{E}, \tilde{F})$. The face set is invariant under mutation $\tilde{F}=F$, and therefore, it is easiest to describe the 


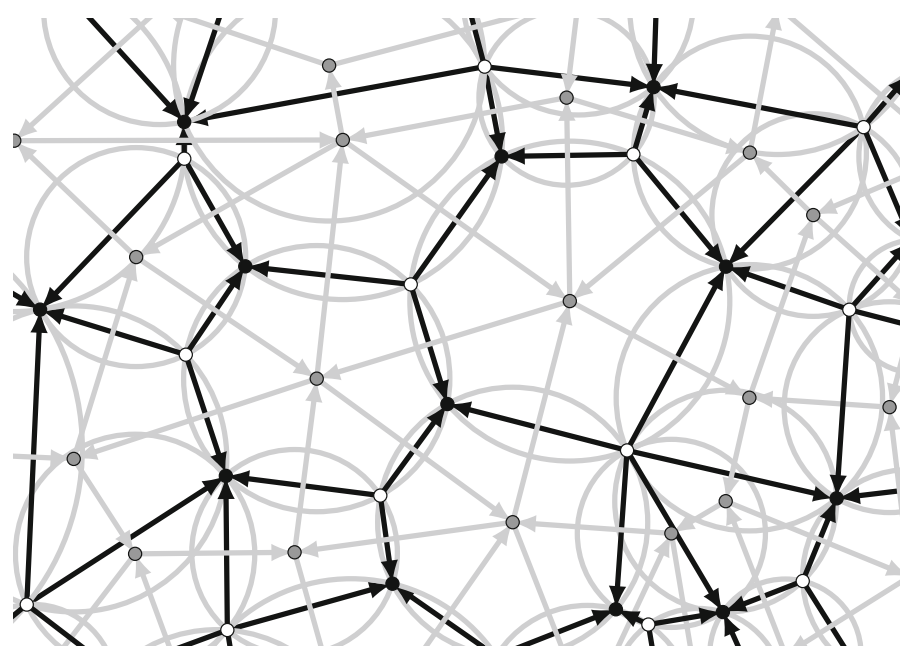

Fig. 1 An excerpt of a circle pattern $z \in \hat{\mathbb{C}}_{\mathrm{o}}^{G}, G \in \mathcal{G}$. The vertices in $V^{+}\left(V^{-}\right)$are colored black (white), and the circle centers which correspond to faces of $G$ are colored gray. Edges of $G$ are drawn with black arrows and edges of $G^{*}$ with gray arrows
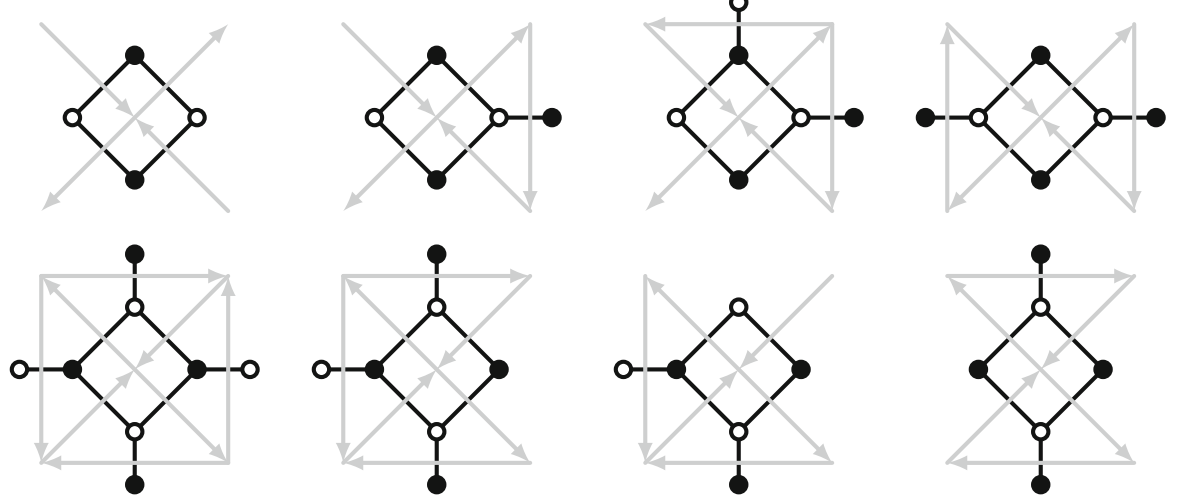

Fig. 2 The local configurations at a face $f$ with four neighbors. The black edges are the edges in $N_{f}$ of the primal graph $G \in \mathcal{G}$. The gray arrows are the edges of the dual graph $G^{*}$. In each column the bottom configuration is the mutation of the upper one and vice versa. Under mutation the set of faces of $G$ is preserved, while the set of vertices changes. The boundary vertices of the above configurations are identified before and after mutation

change in combinatorics with respect to the dual of $G$ :

$$
\tilde{E}^{*}=E^{*} \Delta\left\{\left(f_{1}, f_{2}\right),\left(f_{3}, f_{2}\right),\left(f_{3}, f_{4}\right),\left(f_{4}, f_{1}\right)\right\}
$$

where $\Delta$ denotes the symmetric difference operator. The mutation is an involution: $G=\operatorname{mut}_{f} \tilde{G}$.

Figure 2 shows the possible local configurations and their relation by mutation. The term mutation is borrowed from the theory of cluster algebras, where the 4-mutation 
corresponds to the mutation at a degree four vertex. Indeed, both dimer statistics and Miquel dynamics may be formulated in the language of cluster algebras. We refrain from doing so because we do not need to use any results from the theory of cluster algebras in this paper.

\subsection{Urban renewal}

Because mutation at a quadrilateral $f$ only affects the edges in $N_{f}$, the complementary sets $N_{f}^{\mathrm{c}}$ and $\tilde{N}_{f}^{\mathrm{c}}$ can be identified. Therefore, we are able to compare a matching $M_{0} \in \mathcal{M}(G)$ with a matching $M$ from $\mathcal{M}(G)$ or $\mathcal{M}(\tilde{G})$ by comparing their restrictions to $N_{f}^{\mathrm{c}}$. If $M_{0}$ and $M$ agree on $N_{f}^{\mathrm{c}}$, we write $M_{0} \sim_{f} M$.

Definition 2.5 Fix a quadrilateral $f \in F$ and consider two edge weight functions $\omega \in \mathbb{R}_{+}^{E}$ and $\tilde{\omega} \in \mathbb{R}_{+}^{\tilde{E}}$ on the edge sets of $G$ and $\tilde{G}=\operatorname{mut}_{f} G$, respectively. We say they are related by urban renewal at $f$ if the following two conditions are satisfied:

(i) For all $e \in N_{f}^{\mathrm{c}}: \quad \omega(e)=\tilde{\omega}(e)$

(ii) For any fixed matching $M_{0} \in \mathcal{M}(G)$ :

$$
\sum_{\substack{M \in \mathcal{M}(G) \\ M \sim_{f} M_{0}}} P_{\omega}(M)=\sum_{\substack{M \in \mathcal{M}(\tilde{G}) \\ M \sim \sim_{f} M_{0}}} P_{\tilde{\omega}}(M)
$$

Urban renewal was originally defined explicitly for the edge weights of $G$ [10]. Our definition does not uniquely define the edge weights, only the face weights, which is sufficient for our purposes.

Definition 2.6 For a graph $G \in \mathcal{G}$ we define the face weight function $\tau$ as an alternating ratio of the edge weights as follows:

$$
\tau: \mathbb{R}_{+}^{E} \rightarrow \mathbb{R}_{+}^{F}, \quad(\tau(\omega))(f)=\prod_{\substack{e \in E \\ e^{*}=\left(f, f^{\prime}\right)}} \omega(e) \prod_{\substack{e \in E \\ e^{*}=\left(f^{\prime}, f\right)}}(\omega(e))^{-1}
$$

The first product is over the edges whose duals point into $f$ and the second product is over edges whose duals point away from $f$, where we use the orientation of the dual edges described in Definition 2.2.

The next lemma relating urban renewal and face weights is well known [4,10].

Lemma 2.7 Let $G \in \mathcal{G}$ and $f \in F$ be a quadrilateral. Consider two edge weight functions $\omega \in \mathbb{R}_{+}^{E}$ and $\tilde{\omega} \in \mathbb{R}_{+}^{\tilde{E}}$ that agree on all $e \in N_{f}^{c}$. Adopt the following abbreviations: $\tau_{f}=(\tau(\omega))(f)$ and $\tilde{\tau}_{f}=(\tau(\tilde{\omega}))(f)$. Then $\omega$ and $\tilde{\omega}$ are related by urban renewal at $f$ if and only if the face weights $\tau$ before and the face weights $\tilde{\tau}$ after mutation are related as follows: 


$$
\tilde{\tau}_{f^{\prime}}= \begin{cases}\tau_{f^{\prime}}^{-1} & f^{\prime}=f \\ \tau_{f^{\prime}}\left(1+\tau_{f}\right) & \left(f^{\prime}, f\right) \in E \\ \tau_{f^{\prime}}\left(1+\tau_{f}^{-1}\right)^{-1} & \left(f, f^{\prime}\right) \in E \\ \tau_{f^{\prime}} & \text { else }\end{cases}
$$

\subsection{Circle patterns}

Definition 2.8 Let $G$ be a simple graph and $z: V_{G} \rightarrow \hat{\mathbb{C}}$. Then we call $z$ a vertex drawing of $G$ if no two adjacent vertices are mapped to the same point. Similarly, if $G$ is embedded in a surface and $y: F_{G} \rightarrow \hat{\mathbb{C}}$ is a map such that no two adjacent faces are mapped to the same point we call $y$ a face drawing of $G$.

We work with circles in $\hat{\mathbb{C}}=\mathbb{C} \cup\{\infty\}$, which encompass both the Euclidean circles and straight lines. We call the image of $\infty$ under the inversion in a circle the circle center. Therefore, the center of a straight line is $\infty$.

Definition 2.9 Let $z$ be a vertex drawing of $G \in \mathcal{G}$. We call $z$ a circle pattern if the following two conditions are fulfilled:

(i) For each face $f \in F$, the vertices incident to $f$ are mapped to a common circle in $\hat{\mathbb{C}}$. This allows us to define a map $z^{*} \in \hat{\mathbb{C}}^{F}$ that maps each face of $G$ to the corresponding circle center in $\hat{\mathbb{C}}$.

(ii) $z^{*}$ is a face drawing of $G^{*}$, i.e., circles of neighboring faces have different centers. We denote the set of circle patterns of $G$ by $\hat{\mathbb{C}}_{\circ}^{G}$.

For an example of a circle pattern see Fig. 1.

Definition 2.10 Let $G \in \mathcal{G}$, then the star-ratio sr is a map from the set of face drawings to $\hat{\mathbb{C}}^{F}$ :

$$
\mathrm{sr}: \hat{\mathbb{C}}^{F} \rightarrow \hat{\mathbb{C}}^{F}, \quad(\operatorname{sr}(y))(f)=-\frac{\prod_{\left(f^{\prime}, f\right) \in E}\left(y\left(f^{\prime}\right)-y(f)\right)}{\prod_{\left(f, f^{\prime}\right) \in E}\left(y\left(f^{\prime}\right)-y(f)\right)}
$$

For computations we will also sometimes write:

$$
\operatorname{sr}\left(y ; y_{1}, y_{2}, y_{3}, y_{4}\right)=-\frac{\left(y_{1}-y\right)\left(y_{3}-y\right)}{\left(y_{2}-y\right)\left(y_{4}-y\right)}
$$

where $y, y_{1}, y_{2}, y_{3}, y_{4}$ are all in $\hat{\mathbb{C}}$.

In this definition infinities and zeros cancel and division by zero yields infinity and vice versa.

Lemma 2.11 Given $G \in \mathcal{G}$ and a circle pattern $z \in \hat{\mathbb{C}}_{\circ}^{G}$, the star-ratios of the dual drawing $z^{*} \in \hat{\mathbb{C}}^{F}$ are real. That is:

$$
\forall f \in F: \quad\left(\operatorname{sr}\left(z^{*}\right)\right)(f) \in \mathbb{R}
$$



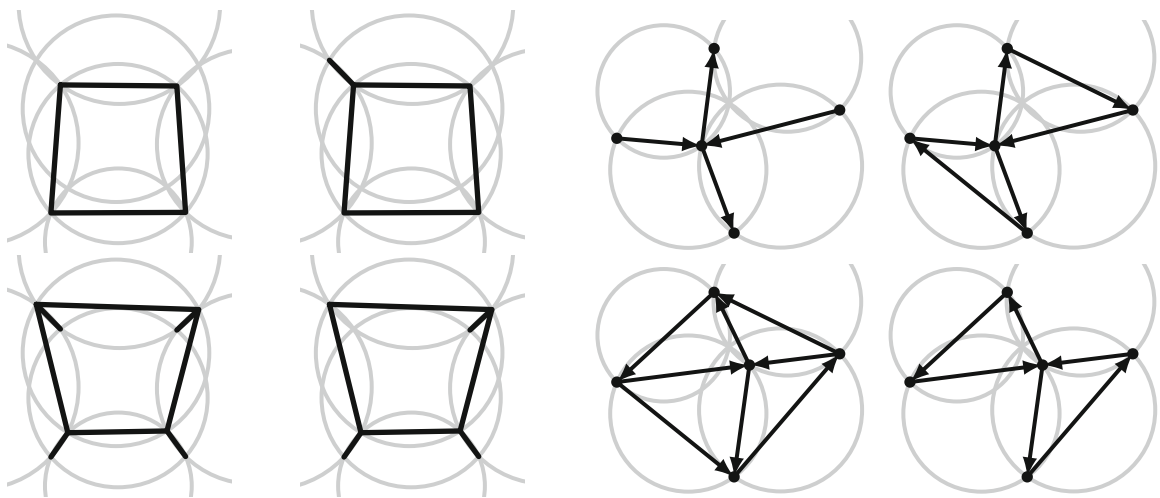

Fig. 3 On the left we have two examples of configurations before (on top) the Miquel move and after (below) the Miquel move. The edges of $G$ are drawn in black, note that the quadrilaterals have a different set of legs, while the circles remain the same. On the right side we see two examples of the Clifford move, where the arrows indicate the oriented dual edges

Proof Observe that the intersection points of $z$ are reflected about the dual edges. The above equation is the closing condition.

Definition 2.12 We call a circle pattern $z \in \hat{\mathbb{C}}_{\circ}^{G}$ of $G \in \mathcal{G}$ Kasteleyn if and only if all the star-ratios of $z^{*} \in \hat{\mathbb{C}}^{F}$ are real positive.

We use the term Kasteleyn because if one uses the complex vectors of the oriented differences between circle centers as weights in an adjacency matrix, this matrix would be Kasteleyn-flat [6,9]. The square root of the determinant of a Kasteleyn-matrix counts weighted perfect matchings.

Definition 2.13 Let $G \in \mathcal{G}$ and $y$ be a face drawing of $G$. Then we call a face $f \in F$ a valid face if the following two conditions are fulfilled:

(i) The face $f$ has exactly four neighbors $f_{1}, f_{2}, f_{3}, f_{4}$, which are listed here in the cyclic order with respect to the orientation of the graph.

(ii) No two consecutive faces are mapped to the same point, that is $y\left(f_{k}\right) \neq y\left(f_{k+1}\right)$ for all four values of $k$, where indices are taken modulo 4 .

We will now introduce the Miquel and the Clifford moves which are based on Miquel's six circle theorem, respectively, Clifford's four circle theorem (see [3]). They locally alter the geometry and are accompanied by a mutation of the combinatorics. For examples see Fig. 3. Miquel's six circle theorem states that given the four circles $c_{1}, c_{2}, c_{3}, c_{4}$ and their four pairs of intersection points $\left\{I_{k}, \tilde{I}_{k}\right\}=c_{k} \cap c_{k+1}$, then: If the four points $I_{1}, I_{2}, I_{3}, I_{4}$ are on a circle $c$, then $\tilde{I}_{1}, \tilde{I}_{2}, \tilde{I}_{3}, \tilde{I}_{4}$ are on a circle $\tilde{c}$. The Miquel move replaces the circle $c$ with the circle $\tilde{c}$. For the definition of the Miquel move, denote the center of $c$ by $M$ and the center of $\tilde{c}$ by $\tilde{M}$.

Definition 2.14 Let $G \in \mathcal{G}, z \in \hat{\mathbb{C}}_{\circ}^{G}$ be a circle pattern and $f \in F$ a valid face with the four neighbors $f_{1}, f_{2}, f_{3}, f_{4}$, such that $z^{*}$ does not map the five faces $f, f_{1}, f_{2}, f_{3}, f_{3}$ 
to a common line. Let $v_{1}, v_{2}, v_{3}, v_{4}$ be the four vertices incident to $f$. Identify $z^{*}(f)=$ $M$ with the center of circle $c$ in Miquel's six circle theorem. Also identify $z\left(v_{k}\right)$ with $I_{k}$. Then the Miquel move replaces the circle associated with $f$ with the alternate one that exists due to Miquel's theorem and yields a new circle pattern on $\tilde{G}=\operatorname{mut}_{f} G$ :

$$
\begin{aligned}
& \operatorname{miq}_{f}: \hat{\mathbb{C}}_{\circ}^{G} \rightarrow \hat{\mathbb{C}}_{\circ}^{\tilde{G}},\left(\operatorname{miq}_{f}(z)\right)\left(v^{\prime}\right)= \begin{cases}\tilde{I}_{k} & v^{\prime}=v_{k} \\
z\left(v^{\prime}\right) & v^{\prime} \neq v\end{cases} \\
& \operatorname{miq}_{f}: \hat{\mathbb{C}}_{\circ}^{F} \rightarrow \hat{\mathbb{C}}_{\circ}^{\tilde{F}}, \quad\left(\operatorname{miq}_{f}\left(z^{*}\right)\right)\left(f^{\prime}\right)= \begin{cases}\tilde{M} & f^{\prime}=f \\
z^{*}\left(f^{\prime}\right) & f^{\prime} \neq f\end{cases}
\end{aligned}
$$

Clifford's four circle theorem considers circles $c_{1}, c_{2}, c_{3}, c_{4}$ that intersect in one common point $I$. Let $J_{k, k+1}$ denote the other intersection points such that $\left\{I, J_{k, k+1}\right\}=c_{k} \cap c_{k+1}$. There are two more intersection points $J_{k, k+2}=c_{k} \cap c_{k+2}$. Also let $\tilde{c}_{k}$ be the circle through the points $J_{k, k+1}, J_{k-1, k}, J_{k-1, k+1}$. Clifford's four circle theorem states that all four circles $\tilde{c}_{k}$ intersect in one point $\tilde{I}$.

Definition 2.15 Let $G \in \mathcal{G}$ be a graph and $y \in \hat{\mathbb{C}}^{F}$ be a face drawing of $G$ and $f \in F$ a valid face with the four neighbors $f_{1}, f_{2}, f_{3}, f_{4}$, such that $y$ does not map the five points $f, f_{1}, f_{2}, f_{3}, f_{3}$ to a common circle. Identify $y(f)=I$ and $y\left(f_{k}\right)=J_{k, k+1}$ with the intersection points in Clifford's four circle theorem above, then set $\left(\operatorname{cli}_{f}(y)\right)(f)=\tilde{I}$. This defines the Clifford move, which takes a face drawing of $G$ to a face drawing of $\tilde{G}=\operatorname{mut}_{f} G$.

$$
\operatorname{cli}_{f}: \hat{\mathbb{C}}^{F} \rightarrow \hat{\mathbb{C}}^{\tilde{F}}: \quad\left(\operatorname{cli}_{f}(y)\right)\left(f^{\prime}\right)= \begin{cases}\tilde{I} & f^{\prime}=f \\ y\left(f^{\prime}\right) & f^{\prime} \neq f\end{cases}
$$

Later on we will show that both the Miquel and the Clifford move are induced by a unique Möbius map. This map can be used to continue the definition of both moves to the degenerate cases which we excluded in the corresponding definitions above.

\section{Main theorems}

Theorem 3.1 Let $G \in \mathcal{G}$ be a graph, let $z \in \hat{\mathbb{C}}_{\circ}^{G}$ be a circle pattern of $G$ and let $z^{*} \in \hat{\mathbb{C}}^{F}$ be the dual drawing induced by the circle centers of $z$. Let $f \in F$ be a valid face. Then the Miquel move at $f$ acts on the circle pattern such that the circle centers change as they do under the Clifford move:

$$
\left(\operatorname{miq}_{f}(z)\right)^{*}=\operatorname{cli}_{f}\left(z^{*}\right)
$$

It is an implication of this theorem that the change of the circle centers does not depend on a particular choice of circles with these circle centers. That is, if $z_{1}, z_{2} \in \hat{\mathbb{C}}_{\circ}^{G}$ are such that $z_{1}^{*}=z_{2}^{*}$, then:

$$
\left(\operatorname{miq}_{f}\left(z_{1}\right)\right)^{*}=\left(\operatorname{miq}_{f}\left(z_{2}\right)\right)^{*}
$$


Corollary 3.2 Consider a Clifford lattice as defined by Konopelchenko and Schief [8]. If one $\mathbb{Z}^{2}$ slice of that lattice has only real star-ratios, then its dynamics is locally governed by the Miquel move.

We will explain this corollary in more detail in Sect. 7.

Definition 3.3 Given a graph $G \in \mathcal{G}$, the map $\psi: \hat{\mathbb{C}}_{\circ}^{G} \rightarrow \mathbb{R}_{+}^{E}$ associates edge weights with a circle pattern $z \in \hat{\mathbb{C}}_{\circ}^{G}$ as follows.

$$
\psi: \hat{\mathbb{C}}_{\circ}^{G} \rightarrow \mathbb{R}_{+}^{E}, \quad(\psi(z))(e)=\left|z^{*}(f)-z^{*}\left(f^{\prime}\right)\right| \quad \forall e \in E,\left(f, f^{\prime}\right)=e^{*}
$$

Theorem 3.4 Let $G \in \mathcal{G}$ be a surface graph, $z \in \hat{\mathbb{C}}_{\circ}^{G}$ a Kasteleyn circle pattern and $f \in F$ a valid face with the four neighbors $f_{1}, f_{2}, f_{3}, f_{4}$, such that $z^{*}$ does not map the five points $f, f_{1}, f_{2}, f_{3}, f_{3}$ to a common line. Then the edge weights $\omega=\psi(z) \in \mathbb{R}_{+}^{E}$ and $\tilde{\omega}=\psi\left(\operatorname{miq}_{f} z\right) \in \mathbb{R}_{+}^{\tilde{E}}$ are related by urban renewal at $f$.

We prove Theorem 3.1 by showing that both the Miquel and the Clifford move are given by the same Möbius map. We introduce this map in Sect. 4 and show that it coincides with the Clifford move in Sect. 5 and that it coincides with the Miquel move in Sect. 6. Theorem 3.4 is proven by analyzing the change of star-ratios under the Möbius map in Sect. 4, which coincides with the change of star-ratios under the Miquel move due to Theorem 3.1.

\section{The star-ratio preserving Möbius map}

In this section we introduce Möbius maps that preserve star-ratios. In particular we are interested in the following problem: Given five points $y, y_{1}, y_{2}, y_{3}, y_{4} \in \hat{\mathbb{C}}$, where are all the points $\tilde{y} \in \hat{\mathbb{C}}$ such that the star-ratio of $y, y_{1}, y_{2}, y_{3}, y_{4}$ and $\tilde{y}, y_{1}, y_{2}, y_{3}, y_{4}$ are the same? It turns out that there is at most one such $\tilde{y} \neq y$, and that $\tilde{y}$ can be expressed as a fractional linear transform of $y$ with coefficients consisting of $y_{1}, y_{2}, y_{3}$ and $y_{4}$. This motivates the definition a unique non-trivial Möbius map that takes any $y$ to $\tilde{y}$ for fixed $y_{1}, y_{2}, y_{3}, y_{4}$.

Definition 4.1 Given four points $y_{1}, y_{2}, y_{3}, y_{4}$ such that $y_{k} \neq y_{k+1}$, we define the Möbius mutation map $\operatorname{mob}\left(y_{1}, y_{2}, y_{3}, y_{4}\right)$.

$$
\operatorname{mob}\left(y_{1}, y_{2}, y_{3}, y_{4}\right): \hat{\mathbb{C}} \rightarrow \hat{\mathbb{C}}, \quad y \mapsto \frac{y C_{2}+C_{3}}{y C_{1}-C_{2}}
$$

The coefficients are the following homogeneous polynomials in the four points $y_{k}$ :

$$
\begin{aligned}
& C_{1}=y_{1}-y_{2}+y_{3}-y_{4} \\
& C_{2}=y_{1} y_{3}-y_{2} y_{4} \\
& C_{3}=y_{2} y_{3} y_{4}-y_{1} y_{3} y_{4}+y_{1} y_{2} y_{4}-y_{1} y_{2} y_{3}
\end{aligned}
$$


Fig. 4 The octahedron which is reflected onto itself by the Möbius mutation map $\operatorname{mob}\left(y_{1}, y_{2}, y_{\overline{1}}, y_{\overline{2}}\right)$

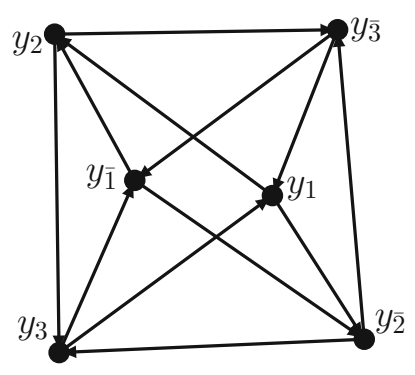

Of course, a fractional linear transform only corresponds to a Möbius map if the determinant given by $-C_{2}^{2}-C_{1} C_{3}$ is not zero. However, the determinant is nonvanishing because it factors as follows:

$$
\operatorname{det} \operatorname{mob}\left(y_{1}, y_{2}, y_{3}, y_{4}\right)=\left(y_{1}-y_{2}\right)\left(y_{2}-y_{3}\right)\left(y_{3}-y_{4}\right)\left(y_{4}-y_{1}\right)
$$

Notice that the trace of the Möbius mutation map is zero, consequently it cannot be the identity. If $y_{1} \neq y_{3}$ or $y_{2} \neq y_{4}$, then the Möbius mutation map is the unique map that maps $y_{k}$ to $y_{k+2}$. It is straightforward to verify that the map interchanges the points as stated. Because we have prescribed the image of at least three points, the map is also unique. If $y_{1}=y_{3}$ and $y_{2}=y_{4}$, then the mutation map is the unique involution that has $y_{1}$ and $y_{2}$ as fixed points.

Lemma 4.2 Let $y_{1}, y_{2}, y_{3}, y_{4} \in \hat{\mathbb{C}}$ such that $y_{k} \neq y_{k+1}$ and let $y \in \hat{\mathbb{C}}$ be such that $y \neq y_{k}$ for any of the four points. Then the Möbius mutation map and the identity are the unique Möbius maps $M: \hat{\mathbb{C}} \rightarrow \widehat{\mathbb{C}}$ such that the following relation holds:

$$
\frac{\operatorname{sr}\left(y ; y_{1}, y_{2}, y_{3}, y_{4}\right)}{\operatorname{sr}\left(M(y) ; y_{1}, y_{2}, y_{3}, y_{4}\right)}=1
$$

Proof Notice that the quotient of star-ratios is decomposable into cross ratios as follows:

$$
\frac{\operatorname{sr}\left(y ; y_{1}, y_{2}, y_{3}, y_{4}\right)}{\operatorname{sr}\left(M(y) ; y_{1}, y_{2}, y_{3}, y_{4}\right)}=\operatorname{cr}\left(y, y_{1}, M(y), y_{2}\right) \operatorname{cr}\left(y, y_{3}, M(y), y_{4}\right)
$$

Therefore, if $M$ satisfies Eq. (4.6), it will do so also after some other Möbius map has been applied. Hence we can assume that no point is at infinity. In that case, Eq. (4.6) is a quadratic equation for $M(y)$ and a simple calculation shows that indeed $M=\operatorname{mob}\left(y_{1}, y_{2}, y_{3}, y_{4}\right)$ or $M$ is the identity. These are the only possibilities because there are at most two solutions.

Lemma 4.3 Consider the octahedron given by the six points $y_{1}, y_{2}, y_{3}, y_{\overline{1}}, y_{\overline{2}}, y_{\overline{3}} \in \hat{\mathbb{C}}$ such that

$$
y_{\overline{1}}=\operatorname{mob}\left(y_{2}, y_{3}, y_{\overline{2}}, y_{\overline{3}}\right)\left(y_{1}\right)
$$


and define quantities $a_{k}$

$$
a_{k}=\frac{\left(y_{k-1}-y_{k}\right)\left(y_{\overline{k-1}}-y_{k}\right)}{\left(y_{k+1}-y_{k}\right)\left(y_{\overline{k+1}}-y_{k}\right)},
$$

for $k \in\{1,2,3\}$. Then the following relations

$$
a_{k}=a_{\bar{k}}, \quad a_{k+1}=-\left(1+a_{k}\right)^{-1} \quad \text { and } \quad a_{k-1}=-\left(1+a_{k}^{-1}\right)
$$

hold for all $k \in\{1,2,3\}$.

Proof By the definition of the Möbius mutation map we can deduce the following symmetric action of the mutation map on the octahedron.

$$
y_{\bar{k}}=\operatorname{mob}\left(y_{l}, y_{m}, y_{\bar{l}}, y_{\bar{m}}\right)\left(y_{k}\right) \quad \forall k, l, m \in\{1,2,3\}, l \neq m
$$

Indeed, it does not matter which two pairs of opposite points we choose to define the mutation map.

$\operatorname{mob}\left(y_{k}, y_{l}, y_{\bar{k}}, y_{\bar{l}}\right)=\operatorname{mob}\left(y_{m}, y_{n}, y_{\bar{m}}, y_{\bar{n}}\right) \quad \forall k, l, m, n \in\{1,2,3\}, k \neq l, m \neq n$

Using the symmetry a short calculation yields the desired result.

Definition 4.4 Let $G \in \mathcal{G}, y$ a face drawing and let $f \in F$ be a valid face of $G$. Extend the definition of the Möbius mutation map to the setting of surface graphs and mutations as follows:

$$
\begin{aligned}
& \operatorname{mob}_{f}: \hat{\mathbb{C}}^{F} \rightarrow \hat{\mathbb{C}}^{\tilde{F}}, \\
& \left(\operatorname{mob}_{f}(y)\right)\left(f^{\prime}\right)= \begin{cases}\operatorname{mob}\left(y\left(f_{1}\right), y\left(f_{2}\right), y\left(f_{3}\right), y\left(f_{4}\right)\right)(y(f)) & f^{\prime}=f \\
y\left(f^{\prime}\right) & f^{\prime} \neq f\end{cases}
\end{aligned}
$$

Lemma 4.5 Let $G \in \mathcal{G}$, y a face drawing and let $f \in F$ be a valid face. Let the tilde denote the quantities on the mutated graph $\widetilde{G}=$ mut $_{f} G$. Under Möbius mutation, the star-ratios change according to the following relations:

$$
\widetilde{\operatorname{sr}}(\tilde{y})\left(f^{\prime}\right)= \begin{cases}\left(\operatorname{sr}(y)\left(f^{\prime}\right)\right)^{-1} & f^{\prime}=f \\ \operatorname{sr}(y)\left(f^{\prime}\right)(1+\operatorname{sr}(y)(f)) & \left(f^{\prime}, f\right) \in E \\ \operatorname{sr}(y)\left(f^{\prime}\right)\left(1+(\operatorname{sr}(y)(f))^{-1}\right)^{-1} & \left(f, f^{\prime}\right) \in E \\ \operatorname{sr}(y)\left(f^{\prime}\right) & \text { else }\end{cases}
$$

Proof Recall the quantities $a_{k}$ from Lemma 4.3. We can identify $a_{1}$ with the star-ratio at $f$ before mutation, $a_{1}$ with the star-ratio after mutation and $a_{2}, a_{3}$ with the quotients of the star-ratios adjacent to $f$ before and after mutation of the graph $G$. 

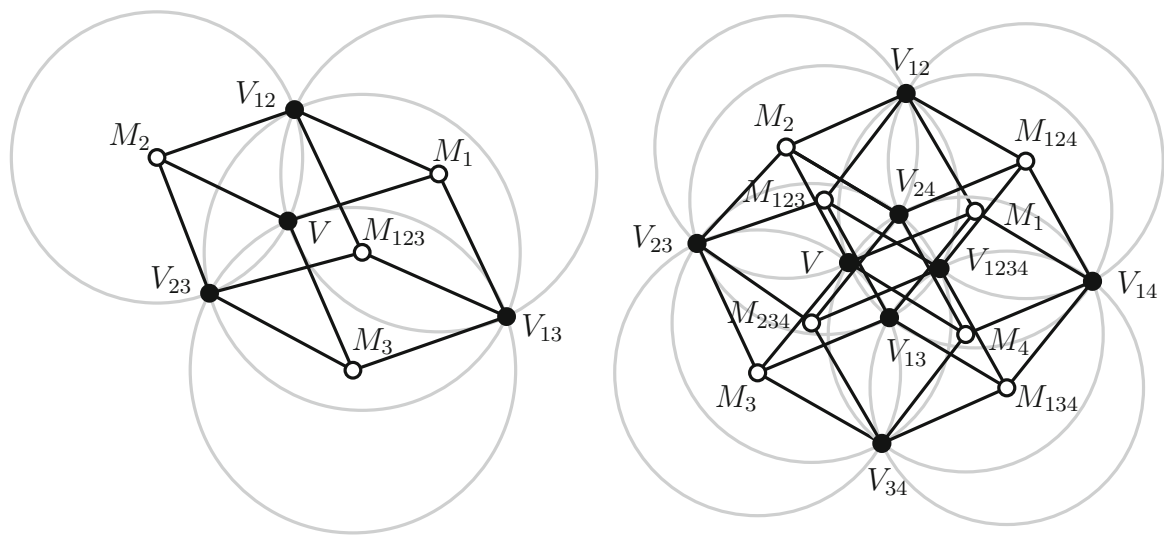

Fig. 5 The Clifford configurations $C_{3}$ and $C_{4}$

We have shown that for four points $y_{1}, y_{2}, y_{3}, y_{4}$ in valid position there exists a unique non-trivial Möbius map that preserves star-ratios with respect to the four points, and we have shown that star-ratios under mutation transform as in Lemma 4.5.

\section{Clifford configurations}

The combinatorial n-hypercube $H_{n}$ has as set of vertices all the subsets of $\{1,2, \ldots, n\}$. There is an edge between $I$ and $I^{\prime}$ in the hypercube if these two sets differ by exactly one index. We call a vertex of the hypercube even (odd) if the cardinality of the corresponding index set is even (odd). The distinction between odd and even sets depends on what vertex has been labeled by the empty set. However, whether two vertices belong to the same parity is independent of that choice.

Definition 5.1 The Clifford-n-circle configuration $C_{n}$ is a map from the n-hypercube $H_{n}$ to points and circles in the plane $\hat{\mathbb{C}}$. Every even vertex is mapped to a point and every odd one to a circle, such that a point incident to a circle in the cube is mapped to that circle in the plane (see Fig. 5 for two examples).

Clifford's theorem then says that given $n$ cyclically ordered circles in the plane all intersecting in one point, they can be extended uniquely to the whole Clifford configuration $C_{n}$. We will denote circles in a Clifford configuration by $c_{I}$, intersection points by $V_{I}$ and circle centers by $M_{I}$. In order to facilitate notation, we will be slightly imprecise and identify the vertices of $H_{n}$ with the corresponding intersection points or circle centers in $\hat{\mathbb{C}}$. The connection of the Clifford configuration $C_{4}$ to the octahedron recurrence and the Möbius mutation map becomes apparent in the next lemma.

Lemma 5.2 Consider the Clifford configuration $C_{4}$. The following two equivalent equalities hold:

$$
\begin{aligned}
\operatorname{mob}\left(V_{12}, V_{23}, V_{34}, V_{14}\right)(V) & =V_{1234} \\
\operatorname{sr}\left(V ; V_{12}, V_{23}, V_{34}, V_{14}\right) & =\operatorname{sr}\left(V_{1234} ; V_{12}, V_{23}, V_{34}, V_{14}\right)
\end{aligned}
$$


Fig. 6 Menelaus' theorem applied to $C_{4}$ with $V_{13}$ sent to infinity via a suitable Möbius transformation

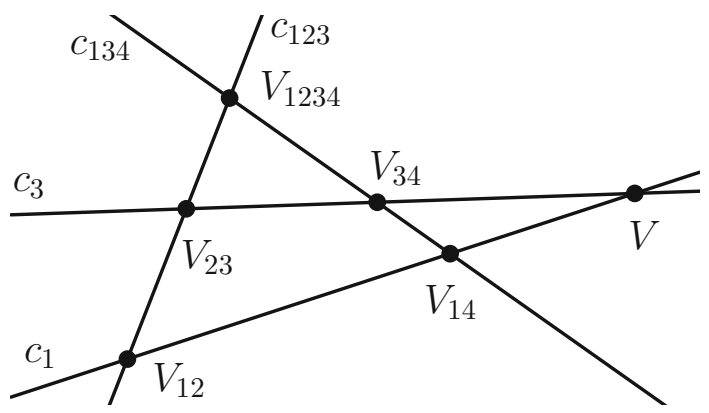

Proof The classical Menelaus' theorem (see [3]) states that given three lines with three distinct intersection points $A_{12}, A_{23}, A_{13}$ and an additional line intersecting the three previous lines in the points $B_{1}, B_{2}, B_{3}$, we have an equality for the multi-ratio of the six points:

$$
\operatorname{mr}\left(A_{12}, B_{2}, A_{23}, B_{3}, A_{13}, B_{1}\right):=\frac{A_{12}-B_{2}}{B_{2}-A_{23}} \frac{A_{23}-B_{3}}{B_{3}-A_{13}} \frac{A_{13}-B_{1}}{B_{1}-A_{12}}=-1
$$

To apply this to $C_{4}$ we send $V_{13}$ to infinity via a Möbius transformation. As a result the circles $c_{1}, c_{3}, c_{123}$ and $c_{134}$ are four lines with the six intersection points $V, V_{12}, V_{14}, V_{23}, V_{34}, V_{1234}$ and of course $V_{13}$ at infinity, compare with Fig. 6 . In this setting Menelaus' theorem gives the following two equalities:

$$
\begin{aligned}
& m_{1}:=\operatorname{mr}\left(V_{34}, V_{14}, V_{1234}, V_{12}, V_{23}, V\right)=-1 \\
& m_{2}:=\operatorname{mr}\left(V_{14}, V_{34}, V_{1234}, V_{23}, V_{12}, V\right)=-1
\end{aligned}
$$

Both $m_{1}$ and $m_{2}$ are actually Möbius invariant quantities as they can be expressed in terms of cross ratios:

$$
\begin{aligned}
& m_{1}=\operatorname{cr}\left(V_{34}, V_{14}, V_{1234}, V_{13}\right) \operatorname{cr}\left(V_{1234}, V_{12}, V_{23}, V_{13}\right) \operatorname{cr}\left(V_{23}, V, V_{34}, V_{13}\right) \\
& m_{2}=\operatorname{cr}\left(V_{14}, V_{34}, V_{1234}, V_{13}\right) \operatorname{cr}\left(V_{1234}, V_{23}, V_{12}, V_{13}\right) \operatorname{cr}\left(V_{12}, V, V_{14}, V_{13}\right)
\end{aligned}
$$

The quotient of star-ratios on the other hand can be expressed by the quantities $m_{1}$ and $m_{2}$

$$
\frac{\operatorname{sr}\left(V ; V_{12}, V_{23}, V_{34}, V_{14}\right)}{\operatorname{sr}\left(V_{1234} ; V_{12}, V_{23}, V_{34}, V_{14}\right)}=\frac{m_{2}}{m_{1}}=1
$$

Because that expression is an equation in cross ratios it is invariant under Möbius transformations and the claim is proven. 
To simplify notation, let us introduce the shift operator $\Delta_{J}$ which acts as a symmetric difference operator on the indices of the arguments of a function, that is:

$$
\begin{aligned}
\Delta_{J} f\left(x_{I_{1}}, x_{I_{2}}, \ldots, x_{I_{m}}\right) & =f\left(x_{J \Delta I_{1}}, x_{J \Delta I_{2}}, \ldots, x_{J \Delta I_{m}}\right) \\
I \Delta J & =(I \cup J) \backslash(I \cap J)
\end{aligned}
$$

Here, the $x$ variables could be vertices $V$, circles $c$ or circle centers $M$. Employing this notation the Möbius mutation map acts as a shift operator on $C_{4}$.

$\operatorname{mob}\left(V_{12}, V_{23}, V_{34}, V_{14}\right)\left(V_{I}\right)=\Delta_{1234} V_{I}, \quad \operatorname{mob}\left(V_{12}, V_{23}, V_{34}, V_{14}\right)\left(c_{I}\right)=\Delta_{1234} c_{I}$

This tells us that $\operatorname{mob}\left(V_{12}, V_{23}, V_{34}, V_{14}\right)$ acts as a total shift on the circles and intersection points of $C_{4}$. However, one should be aware of the fact that the Möbius mutation will in general not act as a shift operator on the circle centers.

In terms of shift notation it is also possible to express the invariance of the star-ratio as follows:

$$
\operatorname{sr}\left(V ; V_{12}, V_{23}, V_{34}, V_{14}\right)=\Delta_{1234} \operatorname{sr}\left(V ; V_{12}, V_{23}, V_{34}, V_{14}\right)
$$

We translate this equality into the following lemma.

Lemma 5.3 Let $G \in \mathcal{G}$ be a graph, y a face drawing of $G$ and let $f \in F$ be a valid face with the four neighbors $f_{1}, f_{2}, f_{3}, f_{4}$, such that $y$ does not map the five points $f, f_{1}, f_{2}, f_{3}, f_{4}$ to a common circle. The Clifford move (see Definition 2.15) coincides with the Möbius mutation map.

$$
\operatorname{cli}_{f}(y)=\operatorname{mob}_{f}(y)
$$

Note that $\operatorname{mob}_{f}(y)$ does not require that the five points $f, f_{1}, f_{2}, f_{3}, f_{4}$ are not on a common circle. Therefore it is natural to use the identity $\operatorname{cli}_{f}(y)=\operatorname{mob}_{f}(y)$ to extend the definition of $\operatorname{cli}_{f}(y)$ also to the case where the five points $f, f_{1}, f_{2}, f_{3}, f_{4}$ are on a common circle. In the next section we prove a similar result for the Miquel move, thereby proving Theorem 3.1 relating Miquel dynamics and Clifford lattices.

In order to make progress later, we will study here a few more cross ratio identities of Clifford configurations. We borrow the following definition from Bobenko, Mercat and Suris [2].

Definition 5.4 Given a map $X: H_{n} \rightarrow \hat{\mathbb{C}}$ from the hypercube $H_{n}$ to the plane, we say it is an integrable cross ratio system if and only if for any $k, a, b \in\{1,2, \ldots, n\}, I \subset$ $\{1,2, \ldots, n\}$ :

$$
\Delta_{k} \operatorname{cr}\left(X_{I}, \Delta_{a} X_{I}, \Delta_{a b} X_{I}, \Delta_{b} X_{I}\right)=\operatorname{cr}\left(X_{I}, \Delta_{a} X_{I}, \Delta_{a b} X_{I}, \Delta_{b} X_{I}\right)
$$

That is, on any 3-cube in $H_{n}$ the cross ratios on opposite 2-faces are the same. 
While this definition does not consider circle centers or intersection points, it can be applied to Clifford configurations and circle patterns.

Lemma 5.5 The $C_{n}$ configuration is an integrable cross ratio system.

Proof A 3-cube in $C_{n}$ is simply $C_{3}$ (see Fig. 5) and can be interpreted as an ideal hyperbolic tetrahedron in the Poincaré half-space model (see [1] for a reference on hyperbolic geometry). The circles represent the ideal boundary of the four involved planes and the intersection points are the four ideal vertices of the tetrahedron. The cross ratio $\operatorname{cr}\left(V, M_{1}, V_{12}, M_{2}\right)$ has absolute value 1 , and its argument is twice the intersection angles of the circles $c_{1}$ and $c_{2}$. Also, it is well known that intersection angles on opposite edges in ideal tetrahedra sum to $\pi$. Thus,

$$
\begin{array}{ll} 
& \operatorname{cr}\left(V, M_{1}, V_{12}, M_{2}\right) \operatorname{cr}\left(V_{13}, M_{123}, V_{23}, M_{3}\right)=1 \\
\Rightarrow \quad & \operatorname{cr}\left(V, M_{1}, V_{12}, M_{2}\right)=\operatorname{cr}\left(M_{3}, V_{13}, M_{123}, V_{23}\right)=\Delta_{3} \operatorname{cr}\left(V, M_{1}, V_{12}, M_{2}\right)
\end{array}
$$

Due to symmetry, this argument works for any cross ratios on pairs of opposite faces in $C_{3}$.

Lemma 5.6 In $C_{4}$ we have that

$$
\operatorname{cr}\left(V_{I}, \Delta_{a b} V_{I}, \Delta_{b c} V_{I}, \Delta_{a c} V_{I}\right)=\Delta_{k} \operatorname{cr}\left(V_{I}, \Delta_{a b} V_{I}, \Delta_{b c} V_{I}, \Delta_{a c} V_{I}\right)
$$

for any $a, b, c, k \in\{1,2,3,4\}, I \subset\{1,2,3,4\}$.

Proof We are comparing two $C_{3}$ configurations. As in Lemma 5.5, the two $C_{3}$ configurations correspond to two hyperbolic tetrahedra, which we denote by $H$ and $\Delta_{k} H$. Also due to Lemma 5.5, we have that

$$
\operatorname{cr}\left(V_{I}, \Delta_{i} V_{I}, \Delta_{j} V_{I}, \Delta_{i j} V_{I}\right)=\Delta_{k} \operatorname{cr}\left(V_{I}, \Delta_{i} V_{I}, \Delta_{j} V_{I}, \Delta_{i j} V_{I}\right)
$$

holds for any $i \neq j$ with $i, j \in\{a, b, c\}$. The occurring cross ratios equal intersection angles of $H$ and $\Delta_{k} H$. The equality states that $H$ and $\Delta_{3} H$ are two hyperbolic tetrahedra with the same intersection angles. It is well known [1] that if two hyperbolic tetrahedra have the same intersection angles, there exists a hyperbolic isometry, which is a Möbius transformation of $\hat{\mathbb{C}}$, that maps one of the tetrahedra onto the other. Because Möbius transformations do not change cross ratios, we obtain that

$$
\operatorname{cr}\left(V_{I}, \Delta_{a b} V_{I}, \Delta_{b c} V_{I}, \Delta_{a c} V_{I}\right)=\Delta_{1234} \operatorname{cr}\left(V_{I}, \Delta_{a b} V_{I}, \Delta_{b c} V_{I}, \Delta_{a c} V_{I}\right)
$$

holds for $a, b, c, I$ as in the lemma. The cross ratio in the right-hand side of the lemma is the cross ratio of the centers of the tetrahedron $\Delta_{3} H$. Therefore it remains to show that

$$
\Delta_{1234} \operatorname{cr}\left(V_{I}, \Delta_{a b} V_{I}, \Delta_{b c} V_{I}, \Delta_{a c} V_{I}\right)=\Delta_{k} \operatorname{cr}\left(V_{I}, \Delta_{i} V_{I}, \Delta_{j} V_{I}, \Delta_{i j} V_{I}\right)
$$


holds. In fact, a short calculation shows that in any $C_{3}$ configuration

$$
\begin{aligned}
\operatorname{mob}\left(\Delta_{a} V_{I}, \Delta_{b} V_{I}, \Delta_{b c} V_{I}, \Delta_{a c} V_{I}\right)\left(V_{I}\right) & =\Delta_{a b c} V_{I} \\
\operatorname{mob}\left(\Delta_{a} V_{I}, \Delta_{b} V_{I}, \Delta_{b c} V_{I}, \Delta_{a c} V_{I}\right)\left(\Delta_{c} V_{I}\right) & =\Delta_{a b} V_{I}
\end{aligned}
$$

holds. Therefore there is a Möbius transformation that maps the four intersection points of a tetrahedron onto its four circle centers and therefore the cross ratio identity (5.20) is proven as well.

Note that even though circle centers are not invariant under Möbius transformations, the previous lemma does indeed relate cross ratios on intersection points of a $C_{3}$ configuration to the cross ratios of circle centers of that same $C_{3}$ configuration. This allows us to prove in the next lemma that star-ratios of circle centers are also preserved under the total shift $\Delta_{1234}$.

Lemma 5.7 Assume a $C_{4}$ configuration, where we picked the origin of the hypercube at the circle center $M$. The following relation holds:

$$
\operatorname{sr}\left(M ; M_{12}, M_{23}, M_{34}, M_{14}\right)=\Delta_{1234} \operatorname{sr}\left(M ; M_{12}, M_{23}, M_{34}, M_{14}\right)
$$

Proof First, we check that this is true for the argument

$$
\arg \operatorname{sr}\left(M ; M_{12}, M_{23}, M_{34}, M_{14}\right)=\arg \operatorname{cr}\left(V_{1}, V_{2}, V_{3}, V_{4}\right)
$$

because each differences appearing in the cross ratio on the right hand side is orthogonal to a difference on the left hand side, thus contributing a factor $\pm i$ each. If the cross ratio is negative then all factors carry the same sign and else two are negative and two positive. Moreover

$$
\operatorname{cr}\left(V_{1}, V_{2}, V_{3}, V_{4}\right)=\Delta_{1234} \operatorname{cr}\left(V_{1}, V_{2}, V_{3}, V_{4}\right)
$$

holds, as $\Delta_{1234}$ corresponds to the action of an involution on the intersection points of $C_{4}$. Finally,

$$
\arg \Delta_{1234} \operatorname{cr}\left(V_{1}, V_{2}, V_{3}, V_{4}\right)=\arg \Delta_{1234} \operatorname{sr}\left(M ; M_{12}, M_{23}, M_{34}, M_{14}\right)
$$

holds for the same reason as in the case without the shift above, and therefore we have shown that

$$
\arg \operatorname{sr}\left(M ; M_{12}, M_{23}, M_{34}, M_{14}\right)=\arg \Delta_{1234} \operatorname{sr}\left(M ; M_{12}, M_{23}, M_{34}, M_{14}\right)
$$


is true. Thus it suffices to check that the quotient of the squares is equal to one. Call this quotient $S^{2}$ and decompose it into cross ratios of circle centers.

$$
\begin{aligned}
S^{2}:= & \left(\frac{\operatorname{sr}\left(M ; M_{12}, M_{23}, M_{34}, M_{14}\right)}{\operatorname{sr}\left(M_{1234} ; M_{12}, M_{23}, M_{34}, M_{14}\right)}\right)^{2} \\
= & \frac{\operatorname{cr}\left(M, M_{12}, M_{13}, M_{23}\right)}{\operatorname{cr}\left(M_{1234}, M_{34}, M_{13}, M_{23}\right)} \frac{\operatorname{cr}\left(M, M_{34}, M_{13}, M_{14}\right)}{\operatorname{cr}\left(M_{1234}, M_{12}, M_{13}, M_{14}\right)} \\
& \cdot \frac{\operatorname{cr}\left(M, M_{34}, M_{24}, M_{23}\right)}{\operatorname{cr}\left(M_{1234}, M_{34}, M_{24}, M_{14}\right)} \frac{\operatorname{cr}\left(M, M_{12}, M_{24}, M_{14}\right)}{\operatorname{cr}\left(M_{1234}, M_{12}, M_{24}, M_{23}\right)}
\end{aligned}
$$

Notice that each cross ratio is a cross ratio of a $C_{3}$ configuration, therefore we are able to apply Lemma 5.6. We shift each cross ratio such that we arrive at an expression in which all the cross ratios cancel.

$$
S^{2}=\frac{\operatorname{cr}\left(V_{123}, V_{3}, V_{2}, V_{1}\right)}{\operatorname{cr}\left(V_{124}, V_{4}, V_{1}, V_{2}\right)} \frac{\operatorname{cr}\left(V_{134}, V_{1}, V_{4}, V_{3}\right)}{\operatorname{cr}\left(V_{234}, V_{2}, V_{3}, V_{4}\right)} \frac{\operatorname{cr}\left(V_{234}, V_{2}, V_{3}, V_{4}\right)}{\operatorname{cr}\left(V_{123}, V_{3}, V_{2}, V_{1}\right)} \frac{\operatorname{cr}\left(V_{124}, V_{4}, V_{1}, V_{2}\right)}{\operatorname{cr}\left(V_{134}, V_{1}, V_{4}, V_{3}\right)}=1
$$

For the special case that the circles around a quadrilateral are part of a $C_{4}$ configuration, Lemma 5.7 already proves the equivalence of the Miquel move and Clifford move on the circle centers. In particular, the so-called integrable circle patterns [2] consist of $C_{n}$ configurations at all faces and vertices. As a result, both the Miquel move and the Clifford move map integrable circle patterns to integrable circle patterns. We will prove in Sect. 6 that locally, it is possible to reduce the case of general circle patterns to the case of an integrable cross ratio system. This will prove the correspondence between Miquel and Clifford move in general.

\section{Circle patterns and Miquel dynamics}

In this section we prove the two main theorems by applying our previous results to the Miquel move. In Lemma 2.11 we have already proven that circle patterns feature real star-ratios, and now we show that in the case of the sphere and the plane the reverse direction is true as well.

Lemma 6.1 Let $G \in \mathcal{G}$ such that it is a decomposition of the sphere $S^{2}$ or the plane $\mathbb{R}^{2}$. Let $y \in \hat{\mathbb{C}}^{F}$ be a face drawing of $G$ such that all star-ratios of y are real. Then there exists a two real-parameter family of drawings $z \in \hat{\mathbb{C}}_{0}^{G}$ which are circle patterns such that $z^{*}=y$.

Proof Let $v_{0} \in V_{G}$ be a fixed vertex, $z_{0} \in \hat{\mathbb{C}}$ and set $z\left(v_{0}\right)=z_{0}$. We prove that we can extend this uniquely to a circle pattern on all of $G$. If $y=z^{*}$ is given and $z(v)$ is given, then $z\left(v^{\prime}\right)$ is uniquely determined for all $\left(v, v^{\prime}\right) \in E_{G}$ because $z\left(v^{\prime}\right)$ is the reflection of $z(v)$ about the dual edge. As $G$ is connected, there is at most one circle pattern such that $z\left(v_{0}\right)=z_{0}$. The fact that this construction via reflections closes around faces (and therefore all cycles) is equivalent to real star-ratios. 

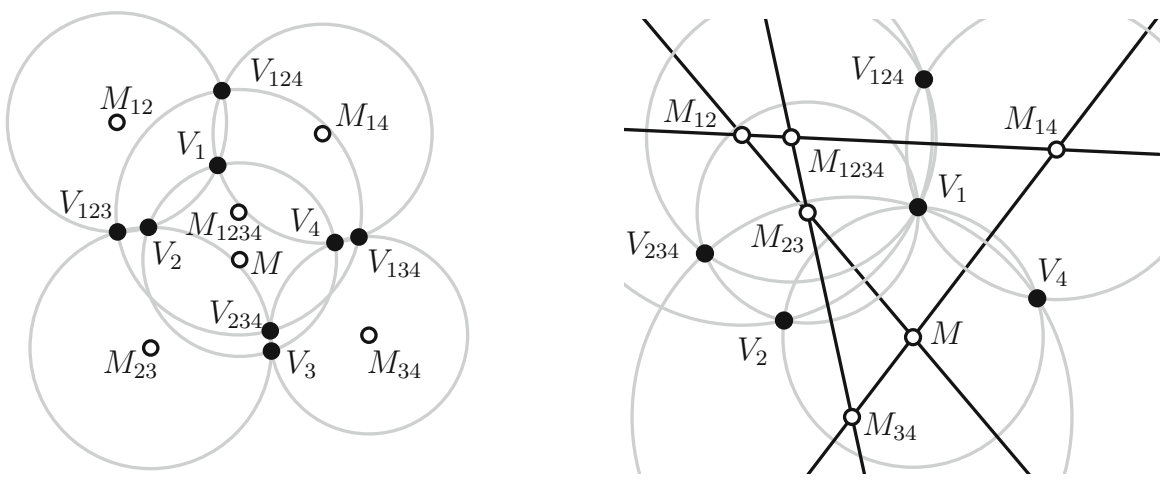

Fig. 7 The general Miquel configuration on the left and the Miquel-Menelaus configuration on the right, where triples of circle centers are on a line

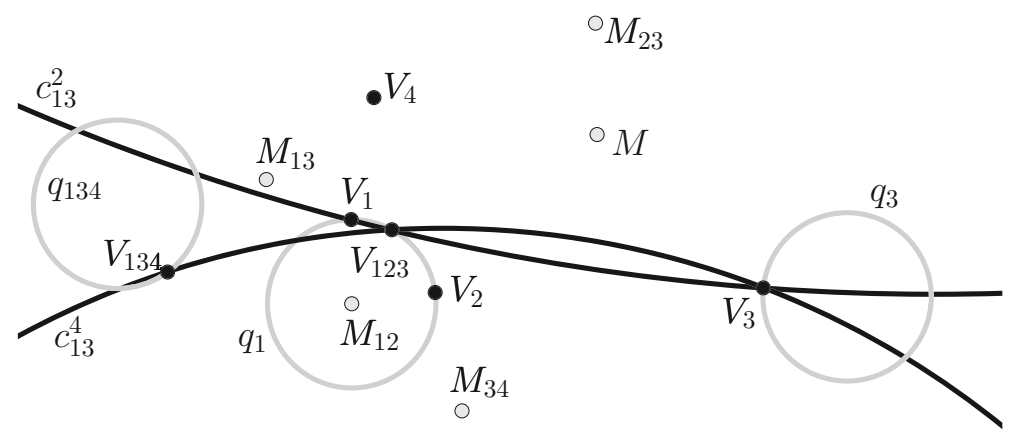

Fig. 8 The Miquel configuration as we use it in the proof of Lemma 6.3, case (ii)

Note that on surfaces like the torus, where cycles exist that are not generated by the face cycles, there need not exist a global circle pattern even if all the star-ratios are real. If it does exist, there will not necessarily be a two real-parameter family of circle patterns either.

In the next step, we show that the action of the Miquel move on the circle centers does not depend on a particular choice of circle pattern with these centers.

Lemma 6.2 If we fix the six circle centers of a Miquel configuration (see Fig. 7), one of the circle intersection points can be chosen arbitrarily in $\hat{\mathbb{C}}$.

Proof The Miquel configuration is a circle pattern on $\mathbb{S}^{2}$. By the previous Lemma 6.1 one intersection point can be chosen freely without changing the circle centers.

Therefore the construction of the sixth circle center via Miquel dynamics does not require a realization by circles, knowing the five other circle centers is actually enough information. The next lemma is the last piece we need to prove our main theorems.

Lemma 6.3 Consider six circles in Miquel's configuration, then:

$$
\operatorname{sr}\left(M ; M_{12}, M_{23}, M_{34}, M_{14}\right)=\operatorname{sr}\left(M_{1234} ; M_{12}, M_{23}, M_{34}, M_{14}\right)
$$


Proof We distinguish two cases:

(i) In the slightly degenerate case that $M$ is on the line $M_{k-1, k} M_{k, k+1}$, we will employ Menelaus' theorem again.

(ii) If that is not the case, we show that there is a choice of circles such that the Miquel configuration is actually a part of a $C_{4}$ configuration.

For the proof of case (i), let us relabel the indices such that $M \in M_{12} M_{23}$, see Fig. 7. The first consequence of $M \in M_{12} M_{23}$ is that $V_{1}=V_{3}$. The second consequence is that thus also $M \in M_{34} M_{14}$. We also note that by definition $V_{134}=V_{123}=V_{1}$. As a result, the perpendicular bisectors to $V_{124}, V_{123}$, respectively, $V_{124}, V_{134}$ both coincide with the line $M_{12} M_{14}$. The same holds for the perpendicular bisectors to $V_{234}, V_{123}$, respectively, $V_{234}, V_{134}$, which are both the line $M_{23} M_{34}$. Finally we have that $M_{1234}=M_{12} M_{14} \cap M_{23} M_{34}$ and we realize that the circle centers are in Menelaus' configuration, see Fig. 7.

In Menelaus' configuration we can use the same argument as in Lemma 5.2 to show that:

$$
\begin{aligned}
\mathrm{mr}_{1} & =\operatorname{mr}\left(M_{1234}, M_{23}, M_{34}, M, M_{14}, M_{12}\right)=-1 \\
\mathrm{mr}_{2} & =\operatorname{mr}\left(M_{1234}, M_{34}, M_{23}, M, M_{12}, M_{14}\right)=-1 \\
\Longrightarrow \quad \frac{\mathrm{mr}_{1}}{\mathrm{mr}_{2}} & =\frac{\operatorname{sr}\left(M ; M_{12}, M_{23}, M_{34}, M_{14}\right)}{\operatorname{sr}\left(M_{1234} ; M_{12}, M_{23}, M_{34}, M_{14}\right)}=1
\end{aligned}
$$

For the proof of case (ii) we show that: Given the six centers $M, M_{12}, M_{23}, M_{34}, M_{14}$, $M_{1234}$ of a Miquel configuration in general position, there is a choice of $V_{1}$ such that the configuration can be extended to a Clifford four circle configuration.

To show that such a choice exists, we introduce a parameter $t \in[0,1]$ moving $V_{1}(t)$ along a small circle $q_{1}$ centered at $M_{12}$ and then use a continuity argument, see also Fig. 8. In particular, we will show that the circles $c_{13}^{2}(t)$ and $c_{13}^{4}(t)$ defined by the point triplets $V_{1}(t), V_{3}(t), V_{123}(t)$ and $V_{1}(t), V_{3}(t), V_{134}(t)$ coincide for some $t_{0}$ and therefore the choice $V_{1}\left(t_{0}\right)$ determines a $C_{4}$ configuration.

Because all the intersection points are determined by reflections about lines given by the circle centers, if $V_{1}(t)$ moves along a circle so do the other intersection points. We also define the circles $q_{3}, q_{123}, q_{134}$ as the trajectories of the points $V_{3}(t), V_{123}(t), V_{134}(t)$. Because $V_{123}(t)$ is given by the successive reflections of $V_{1}(t)$ about the lines $M_{12} M$ and $M_{12} M_{23}$ the circle $q_{123}$ is in fact the same one as $q_{1}$, more so $V_{123}(t)$ is always a constant angle from $V_{1}(t)$ on $q_{1}$. The circles $q_{3}$ and $q_{134}$, on the other hand, cannot coincide with $q_{1}$. Indeed, $q_{3}$ is given by reflections about the lines $M M_{14}$ and $M M_{34}$, while $q_{134}$ is given by reflections about the lines $M M_{14}$ and $M_{14} M_{34}$. Therefore $q_{3}$ or $q_{134}$ can only coincide with $q_{1}$ if $M, M_{14}$ and $M_{34}$ are on a line, which we have treated in case (i). Now assume we choose the radius of $q_{1}$ very small such that from the point of view of $q_{1}$ the circles $q_{3}$ and $q_{134}$ are very far away. In this case at $q_{1}$, the circles $c_{13}^{4}(t)$ look like a 1-parameter family of parallel lines. Therefore it is clear that for very small radius of $q_{1}$, as the point $V_{1}(t)$ winds around $q_{1}$ in counter clockwise direction so does the point $V_{123}(t)$, while the other intersection point of $c_{13}^{4}(t)$ with $q_{1}$ winds around $q_{1}$ in clockwise direction. Therefore there is a $t_{0}$ such that the other intersection point of $c_{13}^{4}(t)$ with $q_{1}$ is $V_{123}\left(t_{0}\right)$. Finally, because 
$c_{13}^{2}\left(t_{0}\right)$ and $c_{13}^{4}\left(t_{0}\right)$ have the three points $V_{1}\left(t_{0}\right), V_{3}\left(t_{0}\right), V_{134}\left(t_{0}\right)$ in common they are in fact the same.

This proves that we can choose the intersection points such that $M, M_{12}, M_{23}, M_{34}$, $M_{14}, M_{1234}$ are centers of a Clifford four circle configuration and thus it proves this case due to Lemma 5.7.

We have now shown that indeed the Miquel move on the circle centers acts as the Möbius mutation does. As in the previous section, let us put this into a lemma.

Lemma 6.4 Let $G \in \mathcal{G}$ be a graph, $z \in \hat{\mathbb{C}}_{0}^{G}$ be a circle pattern and let $f \in F$ be a valid face with the four neighbors $f_{1}, f_{2}, f_{3}, f_{4}$, such that $z^{*}$ does not map the five faces $f, f_{1}, f_{2}, f_{3}, f_{4}$ to a common line. The Miquel move coincides with the Möbius mutation map on the induced face drawing $z^{*}$ :

$$
\left(\operatorname{miq}_{f}(z)\right)^{*}=\operatorname{mob}_{f}\left(z^{*}\right)
$$

As in the case of the Clifford move, it is possible to interpret the Möbius mutation map as the extension of the Miquel move on the circle centers to the degenerate case that all the five centers are on a common line.

We can now proceed to prove the two main theorems. As a short reminder, Theorem 3.1 states that the Miquel and the Clifford move coincide and Theorem 3.4 states that the Miquel move behaves like urban renewal on the dimer model induced by the dual edge lengths.

Proof of Theorem 3.1 Let $z$ be a circle pattern of $G \in \mathcal{G}$ and $f$ be a valid face. In Lemma 5.3 and Lemma 6.4 we have proven the following two equalities:

$$
\begin{aligned}
\operatorname{cli}_{f}\left(z^{*}\right) & =\operatorname{mob}_{f}\left(z^{*}\right) \\
\left.\operatorname{miq}_{f}(z)\right)^{*} & =\operatorname{mob}_{f}\left(z^{*}\right)
\end{aligned}
$$

Together these two equations imply the theorem, namely that:

$$
\operatorname{cli}_{f}\left(z^{*}\right)=\left(\operatorname{miq}_{f}(z)\right)^{*}
$$

Proof of Theorem 3.4 In the case of a Kasteleyn circle pattern $z \in \hat{\mathbb{C}}_{0}^{G}$ all the star-ratios of $z^{*} \in \hat{\mathbb{C}}^{F}$ are real and positive. Therefore in this case, the star-ratios only depend on the absolute values of the differences. Thus, if we consider the dimer edge weights $\psi$ on $G$ given by $z$ as in Definition 3.3, we conclude that:

$$
(\tau(\psi(z)))(f)=\left(\operatorname{sr}\left(z^{*}\right)\right)(f) \quad \forall f \in F
$$

Hence, the star-ratios are indeed the face weights of the associated dimer model. We have proven that the star-ratios transform under the Miquel move as they do under the Möbius mutation map in Lemma 4.5. In the case that the star-ratios are real, these are indeed real formulas as well. We observe that they are exactly those of Lemma 2.7 on the change of face weights $\tau$ in urban renewal. Thus the theorem is proven. 


\section{Lattice dynamics}

Even though the new results of this paper are of local nature, we want to shortly outline the connection to dynamics on lattices. In particular, Miquel dynamics has originally been defined as an evolution of circle patterns on periodic $\mathbb{Z}^{2}$ lattices, and the study of Clifford lattices has taken place on the octahedral lattice, a sublattice of $\mathbb{Z}^{3}$.

Define the parity $|p|$ of a point $p=\left(p_{1}, p_{2}, p_{3}\right) \in \mathbb{Z}^{3}$ as follows:

$$
|\cdot|: \mathbb{Z}^{3} \rightarrow \mathbb{Z}_{2}, \quad|p|=\sum_{k=1}^{3} p_{k} \quad \bmod 2
$$

Definition 7.1 The three-dimensional octahedral lattice $\mathbb{Z}_{0}^{3}$ consists of all points in $\mathbb{Z}^{3}$ that have even parity, together with edges between the nearest neighbors:

$$
\begin{gathered}
V\left(\mathbb{Z}_{0}^{3}\right)=\left\{p \in \mathbb{Z}^{3}|| p \mid=0\right\} \\
E\left(\mathbb{Z}_{0}^{3}\right)=\left\{\left(p, p^{\prime}\right) \in V\left(\mathbb{Z}_{0}^{3}\right) \times V\left(\mathbb{Z}_{0}^{3}\right)\left|\max _{k=1,2,3}\right| p_{k}-p_{k}^{\prime} \mid=1\right\}
\end{gathered}
$$

Define a level slice $S_{k}$ of the octahedral lattice as the vertices with third coordinate in $\{k, k+1\}$ together with the edges of $\mathbb{Z}_{0}^{3}$ that connect two different layers.

$$
\begin{gathered}
V\left(S_{k}\right)=\left\{p \in V\left(\mathbb{Z}_{0}^{3}\right) \mid p_{3} \in\{k, k+1\}\right\} \\
E\left(S_{k}\right)=\left\{\left(p, p^{\prime}\right) \in E\left(\mathbb{Z}_{0}^{3}\right) \mid p, p^{\prime} \in V\left(S_{k}\right) \text { and } p_{3} \neq p_{3}^{\prime}\right\}
\end{gathered}
$$

It is clear that such a level slice $S_{k}$ has the combinatorics of the $\mathbb{Z}^{2}$ lattice. As such, it can be bi-partitioned into the set of points which have $p_{3}=k$ and the set of points which have $p_{3}=k+1$.

$$
\begin{gathered}
S_{k}^{0}:=\left\{p \in V\left(S_{k}\right) \mid p_{3}=k\right\} \\
S_{k}^{+}:=\left\{p \in V\left(S_{k}\right) \mid p_{3}=k+1\right\}
\end{gathered}
$$

It follows that $S_{k}$ and $S_{k+1}$ are two $\mathbb{Z}^{2}$ lattices that as subsets of the octahedral lattice agree on every second point, that is $S_{k}^{+}=S_{k+1}^{0}$.

Set $G=S_{k} \in \mathcal{G}$ considering it as a surface graph. Then the combinatorial 4mutation at every vertex in $S_{k}^{0}$ gives a new graph $G^{\prime}$ isomorphic to $\mathbb{Z}^{2}$ which we can identify with $S_{k+1}$, such that $S_{k}^{+}=S_{k+1}^{0}$ and all the vertices we mutated at are in $S_{k}^{0}$ before mutation and in $S_{k+1}^{+}$after mutation. In that sense mutation replaces a vertex with the vertex two steps into the 3-direction of the octahedral lattice. 
Let $z \in \widehat{\mathbb{C}}^{V_{k}}$ be a drawing where $V_{k}=V\left(S_{k}\right)$. Then by the combinatorial identification above and the Möbius mutation map this drawing determines a unique function $z: V\left(\mathbb{Z}_{0}^{3}\right) \rightarrow \widehat{\mathbb{C}}$ from the octahedral lattice to the complex plane.

$$
z\left(p_{x, y, z+1}\right)=\operatorname{mob}\left(z\left(p_{x+1, y+1, z}\right), z\left(p_{x+1, y-1, z}\right), z\left(p_{x-1, y-1, z}\right), z\left(p_{x-1, y+1, z}\right)\right)\left(z\left(p_{x, y, z-1}\right)\right)
$$

Functions on octahedral lattices which are constructed as above have been characterized as discrete integrable systems in [8]. A system is called discretely integrable if there exists a function on a lattice such that it satisfies a given set of local equations at each point in the lattice.

It is clear from the construction above that this is a 3-d integrable system in the sense that the data that determines the whole system (the Cauchy data) is 2-d, as we can choose freely the function on all points of a given slice in the octahedral lattice. In the case of circle patterns it is clear that we can choose as Cauchy data any circle pattern with the combinatorics of $\mathbb{Z}^{2}$. Such a circle pattern can be constructed row by row. Given a row, each circle center of the next row has to be on the bisector of the two corresponding intersection points of the given row, therefore there is a onedimensional real degree of freedom per circle. If we are interested in Kasteleyn circle patterns, then each circle center has to be chosen on an $\mathbb{R}^{+}$ray and the Cauchy data are therefore still 2-d.

Given fixed star-ratios on $\mathbb{Z}^{2}=S_{k}$, then Lemma 4.5 asserts that we know the star-ratios on all slices $S_{k^{\prime}}$ of the octahedral lattice. However, the knowledge of all the star-ratios on $\mathbb{Z}^{2}$ does not fix the drawing $z \in \hat{\mathbb{C}}^{\mathbb{Z}_{2}}$. Instead, the fixed star-ratios determine a 2-d discrete integrable system themselves. Given the drawing $z$ on a strip $\mathbb{Z} \times\{l, l+1\}$, the star-ratios determine the whole drawing on $\mathbb{Z}^{2}$. This strip is in fact the intersection of two level slices in two different directions. In this case, the prescription of real star-ratios corresponds to the case of circle patterns and the prescription of real and positive star-ratios corresponds to the case of Kasteleyn circle patterns.

An octahedral lattice constructed by the Möbius mutation from given data on $\mathbb{Z}^{2}$ carries additional symmetry: The three space directions of the lattice are indistinguishable. This is a consequence of the symmetric nature of the Möbius mutation map as explained in Sect. 4. In particular, if the star-ratios are the same on the two opposite stars in an octahedron of the lattice, then they are equal on all three pairs of opposite stars in that octahedron, see Lemma 4.3. Moreover, if we prescribe real star-ratios in the given Cauchy data, then all the star-ratios of the whole lattice will be real, see Lemma 4.5. Therefore an octahedral lattice with real star-ratios induces three families of $\mathbb{Z}^{2}$ circle patterns, each family consisting of circle patterns related via Miquel dynamics. However, this symmetry does not hold for Kasteleyn circle patterns. If one level slice $S_{k}$ corresponds to a Kasteleyn circle pattern, then all the parallel slices are Kasteleyn as well, but the slices in the other directions are necessarily not Kasteleyn. This is an immediate consequence of the identities in Lemma 4.3, which show that positive star-ratios in one direction imply negative star-ratios in the other directions.

Acknowledgements The author would like to thank Ananth Sridhar, Boris Springborn, Wolfgang Schief and Jan Techter for discussions and advice. 
Funding Open Access funding enabled and organized by Projekt DEAL. This research was supported by the Deutsche Forschungsgemeinschaft (DFG) Collaborative Research Center TRR 109 "Discretization in Geometry and Dynamics."

\section{Declarations}

Conflict of interest On behalf of all authors, the corresponding author states that there is no conflict of interest.

Open Access This article is licensed under a Creative Commons Attribution 4.0 International License, which permits use, sharing, adaptation, distribution and reproduction in any medium or format, as long as you give appropriate credit to the original author(s) and the source, provide a link to the Creative Commons licence, and indicate if changes were made. The images or other third party material in this article are included in the article's Creative Commons licence, unless indicated otherwise in a credit line to the material. If material is not included in the article's Creative Commons licence and your intended use is not permitted by statutory regulation or exceeds the permitted use, you will need to obtain permission directly from the copyright holder. To view a copy of this licence, visit http://creativecommons.org/licenses/by/4.0/.

\section{References}

1. Alekseevskij, D.V., Vinberg, È.B, Solodovnikov, A.S.: Geometry of spaces of constant curvature. In: Geometry, II, volume 29 of Encyclopaedia Math. Sci., pages 1-138. Springer, Berlin, (1993)

2. Bobenko, Alexander I., Mercat, Christian, Suris, Yuri B.: Linear and nonlinear theories of discrete analytic functions. Integrable structure and isomonodromic Green's function. Journal für die reine und angewandte Mathematik 2005, 117-161 (2005)

3. Bobenko, A.I., Suris, Y.B.: Discrete Differential Geometry: Integrable Structure. Graduate studies in mathematics, Birkhäuser (2008)

4. Goncharov, A.B., Kenyon, R.: Dimers and cluster integrable systems. Ann. Sci. Éc. Norm Supér. 46(5), 747-813 (2013)

5. Glutsyuk, A., Ramassamy, S.: A first integrability result for Miquel dynamics. J. Geom. Phys. 130, 121-129 (2018)

6. Kasteleyn, P.W.: The statistics of dimers on a lattice: I. The number of dimer arrangements on a quadratic lattice. Physica 27(12), 1209-1225 (1961)

7. Kenyon, R., Lam, W.Y., Ramassamy, S., Russkikh, M.: Dimers and Circle patterns. To appear in the Annales Scientifiques de l'ENS. Preprint on arXiv:1810.05616, October (2018)

8. Konopelchenko, B.G., Schief, W.K.: Menelaus' theorem, Clifford configurations and inversive geometry of the Schwarzian KP hierarchy. J. Phys. A Math. Gen. 35(29), 6125 (2002)

9. Kuperberg, Greg: An exploration of the permanent-determinant method. The electronic journal of combinatorics $\mathbf{5}(1)$, (1998)

10. Propp, J.: Generalized domino-shuffling. Theoret. Comput. Sci. 303(2), 267-301 (2003)

11. Ramassamy, Sanjay: Miquel Dynamics for Circle Patterns. Published online, International Mathematics Research Notices (2018)

Publisher's Note Springer Nature remains neutral with regard to jurisdictional claims in published maps and institutional affiliations. 